\title{
Unusual ovarian leukemic relapse in a girl with history of B cell lymphoblastic leukemia
}

\author{
Adrian Chi Heng Fung ${ }^{1}$ and Kenneth Kak Yuen Wong ${ }^{1}$ \\ ${ }^{1}$ University of Hong Kong
}

April 29, 2021

Unusual ovarian leukemic relapse in a girl with history of B cell lymphoblastic leukemia

ACH Fung, KKY Wong

To the editor:

Extramedullary leukemic recurrence mostly occurs in the central nervous system and occasionally in the skin. [1] Here, we report an unusual case of a girl with relapsed lymphoblastic leukemia involving the ovary, presenting as a large pelvic mass.

A 10-year-old girl with history of B cell lymphoblastic leukaemia treatment and in remission 1 year ago presented to oncology clinic with an enlarging pelvic mass for 2 months. Magnetic resonance imaging revealed a large lobulated solid heterogeneous pelvic mass (measuring $12 \mathrm{~cm}$ in greatest dimension) with mass effect on pelvic organs (Figure 1a). Bone marrow aspirate at conventional site confirmed absence of lymphoblastic cells, which would have suggested leukaemia relapse. In view of the suspicion of a second primary tumour in the ovary with complication, exploratory laparotomy and left salpingo-oophorectomy were performed (Figure 1b). Histology showed diffuse infiltration of the ovary by B cell lymphoblastic leukaemia. Hematoxylin and eosin staining showed sheets of lymphoid cells with irregular nuclei. The tumour cells are positive for TdT, CD19, CD79a and CD34. (Figure 1c) She was well after operation. In view of extramedullary relapse, bone marrow aspirate was repeated after operation at anterior iliac spine which confirmed bone marrow relapse. She received treatment according to high risk protocol of CCCG Relapsed Acute Lymphoblastic Leukemia 2017 Study in Children [2].

Acute lymphoblastic leukaemia (ALL) is responsible for one-third of childhood malignancy. Despite efforts in the treatment of ALL, $15-20 \%$ of patients developed relapse with highest risk immediately after cessation of treatment and diminishes with time. [3] Extramedullary involvement constitutes 2-5\% of all relapses with the majority in the central nervous system. It seldom involves other sites, such as the eye, kidney, and omentum. Ovarian leukemic relapse is rarely reported in children with scarce case reports in the literature [4, 5] It is insidious and not usually detected until symptoms arise. Patient most commonly presents with lower abdominal pain or a palpable large abdominal mass. [6] Timely detection is important as it often coincides with marrow recurrence. Routine surveillance pelvic sonography is recommended for timely detection of pelvic extramedullary relapse.[7] In patients with background of leukaemia presenting with an ovarian mass, a higher level of suspicion needs to be kept. In the absence of evidence in bone marrow relapse at conventional site, repeat marrow aspirate at another site would be warranted since this affect the choice of management. Previous reports shown that mainly chemotherapy had a beneficial effect, while neither local radiation nor extensive surgical resection of the leukaemic mass had any obvious effect on overall outcome [8]. Operation could have been avoided if marrow relapse is confirmed at the time of ovarian mass detection. However, surgical excision was reported to have role in situation when bone marrow had good response but no signs of regression of ovarian mass. [9] 


\section{Reference}

1. Kim, J.W., et al., Ovarian and multiple lymph nodes recurrence of acute lymphoblastic leukemia: a case report and review of literature. Pediatr Surg Int, 2008. 24 (11): p. 1269-73.

2. CCCG Relapsed Acute Lymphoblastic Leukemia 2017 Study in Children .https://ClinicalTrials.gov/show/NCT04224571.

3. Berretta, R., et al., Isolated ovarian relapse of pre-B acute lymphoblastic leukemia: a case report. J Pediatr Adolesc Gynecol, 2009.22 (4): p. e65-8.

4. Kantekure, K., et al., A unique case of relapsed B-acute lymphoblastic leukemia/lymphoma as an isolated omental mass. Case Rep Hematol, 2014. 2014 : p. 425163.

5. Sava, C.N., et al., Unusual extramedullary relapses in a case of common B-cell acute lymphoblastic leukemia. Case report and review of literature. Rom J Morphol Embryol, 2019. 60 (1): p. 249-254.

6. Turial, S., et al., Ovarian tumours: late extramedullary recurrence of acute leukaemia. Eur J Pediatr Surg, 2009.19 (3): p. 184-6.

7. Lane, D.M. and R.L. Birdwell, Ovarian leukemia detected by pelvic sonography. A case report. Cancer, 1986. 58 (10): p. 2338-42.

8. Pais, R.C., et al., Ovarian tumors in relapsing acute lymphoblastic leukemia: a review of 23 cases. J Pediatr Surg, 1991.26 (1): p. 70-4.

9. Till, H., O. Muensterer, and U. Graubner, Laparoscopic adnexectomy of a persistent ovarian tumor in a girl with acute lymphoblastic leukemia relapse. Pediatr Hematol Oncol, 2003.20 (5): p. 417-20. 


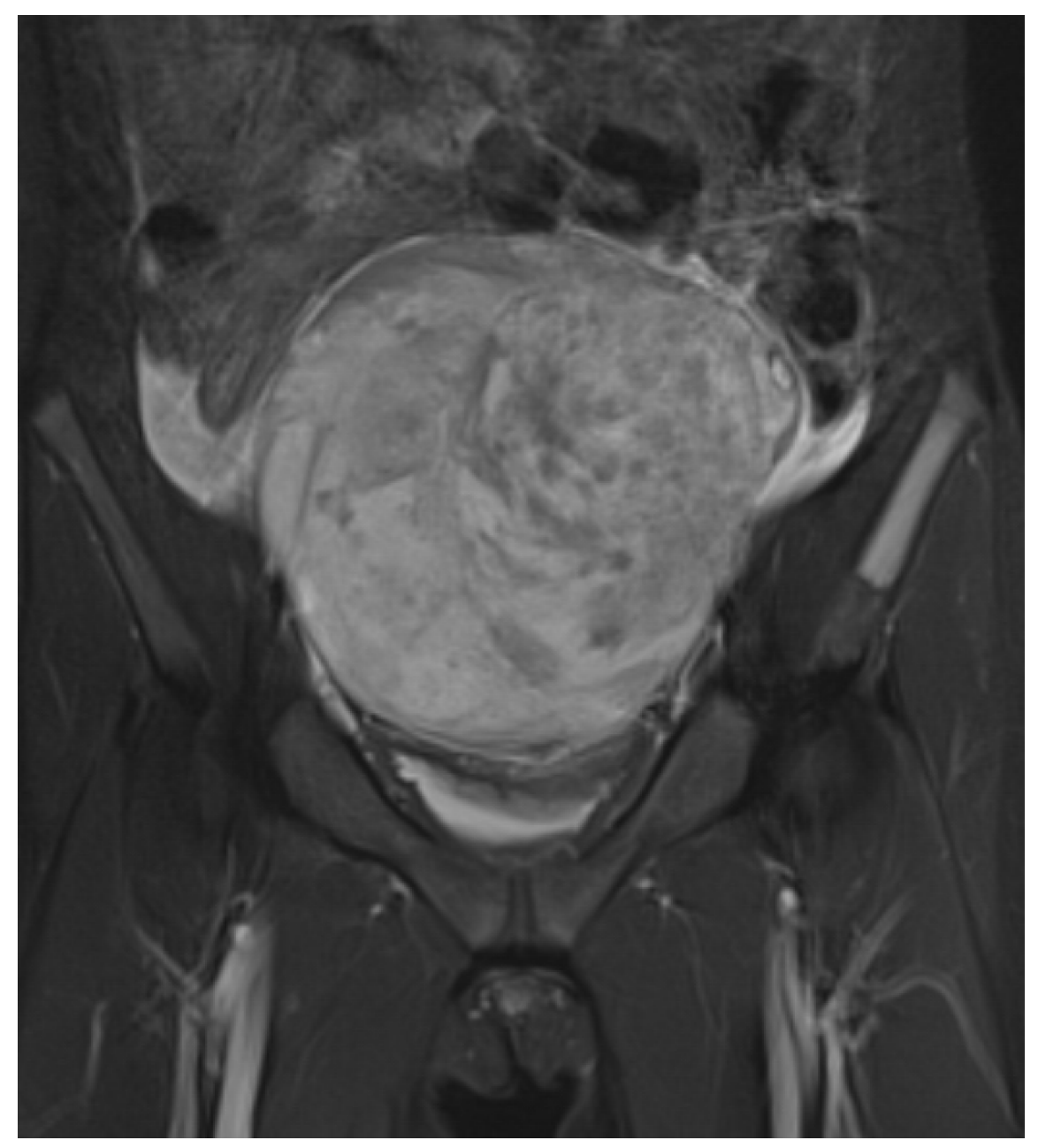

Figure 1a: Magnetic resonance imaging showing a large lobulated solid heterogeneous pelvic mass with mass effect on pelvic organs 


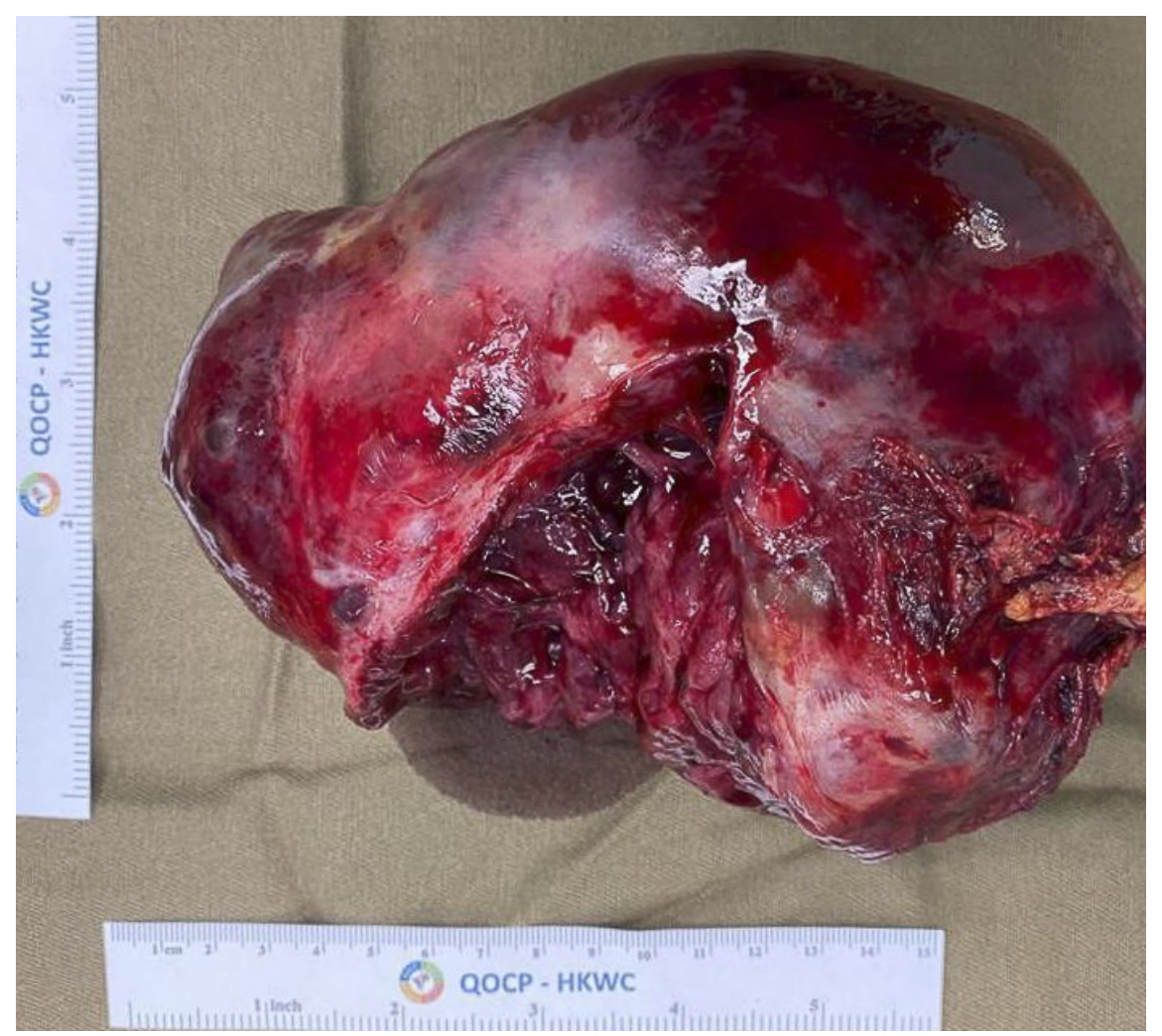

Figure 1b: Surgical specimen of the large ovarian tumor

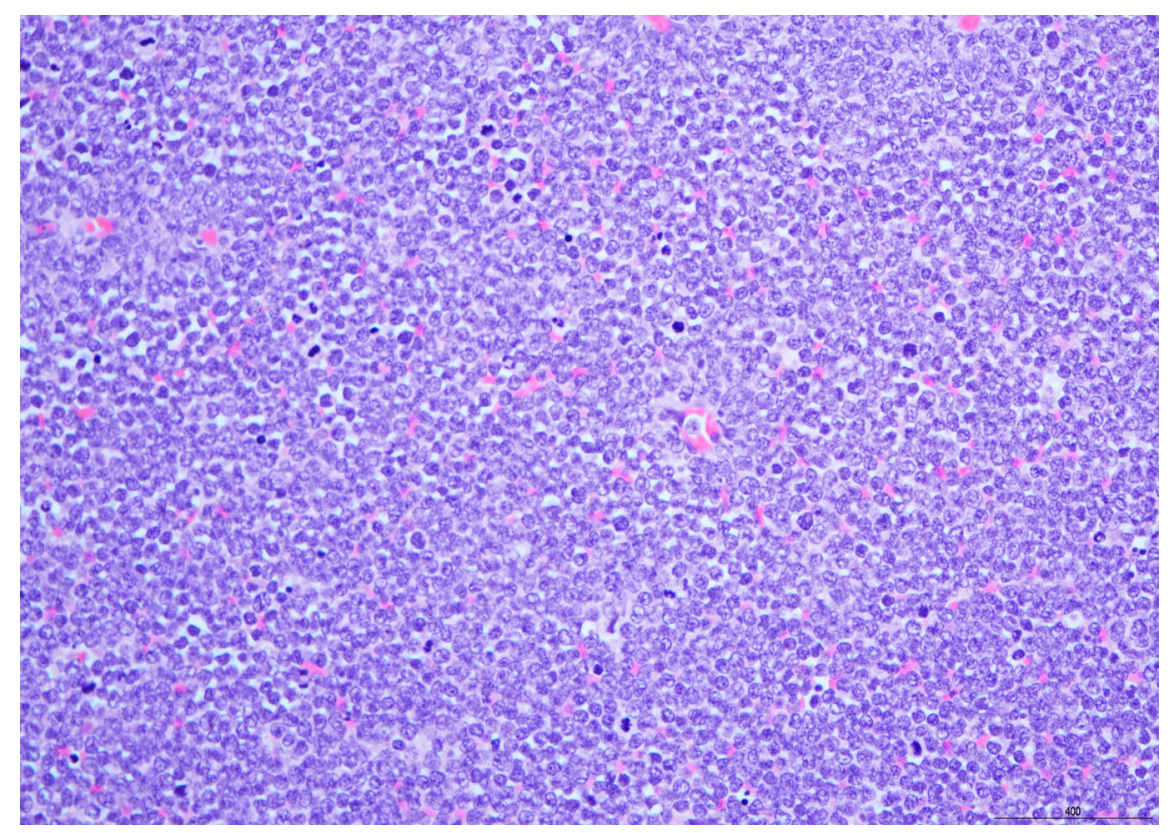

Figure 1c: Histology of ovarian tumor: Diffuse infiltration of the ovary by sheets of small to medium sized lymphoid cells with irregular nuclei that lack nucleoli formation and scanty cytoplasm. (Hematoxylin and 
eosin staining, original magnification x20)

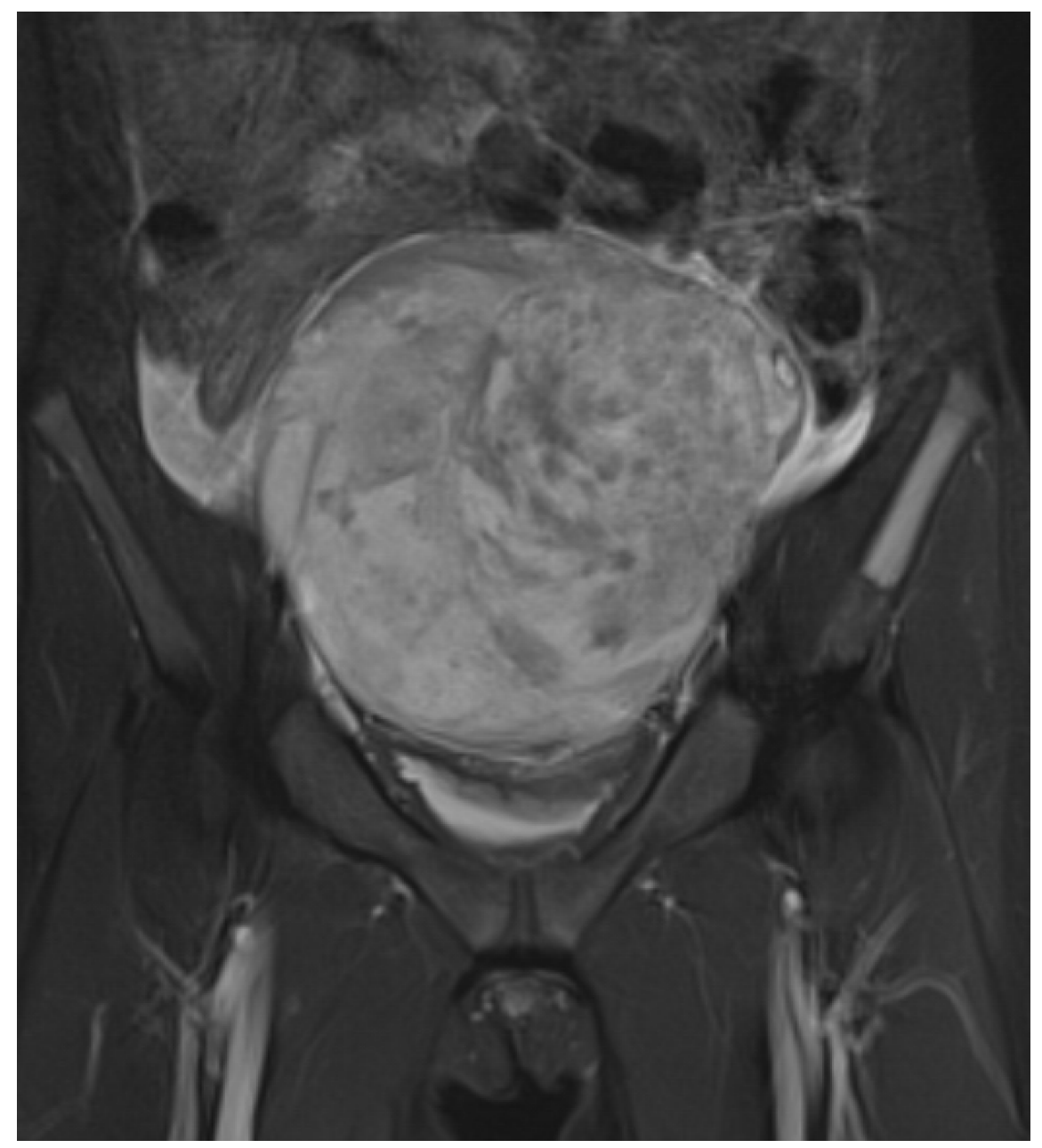



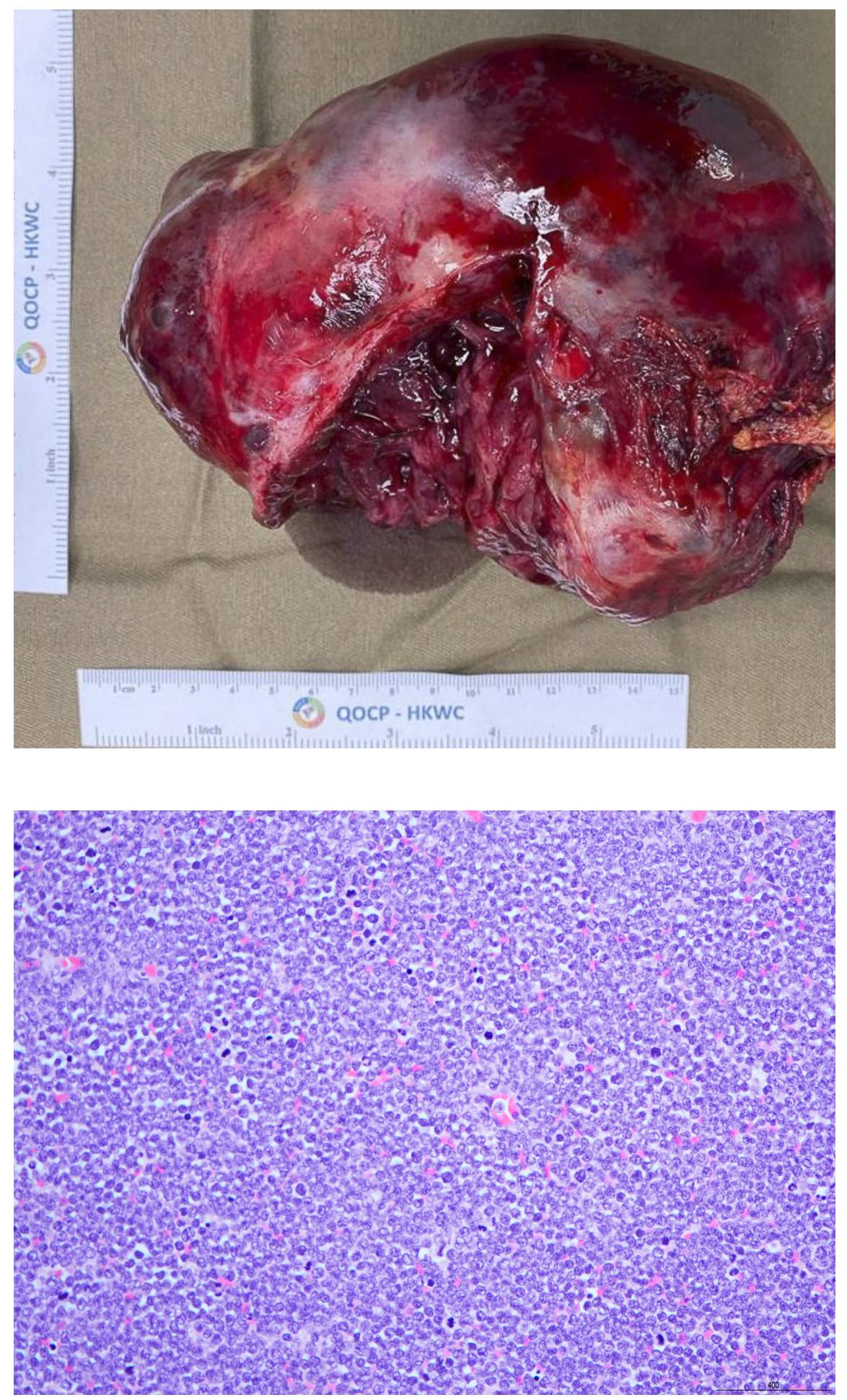